\title{
Ein Satz über Kegelschnitte mit einigen Anwendungen auf die perspektive Affinität.
}

\author{
Fon \\ Fritz Carlson in Upsala.
}

$\S 1$.

Wir betrachten ein Büschel Kegelschnitte $*$ mit den zwei Grundpunkten $a, b$; die anderen beiden mögen in $O$ zusammenfallen, so daß alle $\approx$ eine feste Gerade $O H$ in $O$ berühren. Eine beliebige Gerade $l$ schneidet die Kegelschnitte des Büschels in Punktpaaren einer Involution, deren Doppelpunkte von den zwei $l$ berührenden Kegelschnitten $\varkappa$ geliefert werden. Der Schnittpunkt $H$ der Verbindungsgerade $a b$ mit der Tangente in $O$ hat dieselbe Polare $O \Pi$ in bezug auf alle Kegelschnitte des Büschels; sie liegt zu $O H$ harmonisch bezüglich $O a, O b$. Es seien $x_{s}, x_{t}$ zwei Kegelschnitte im Büschel $*$ und $\sigma_{1} \tau_{1}, \sigma_{9} \tau_{\Xi}$ die gemeinsamen Tangenten, wo $\sigma_{1}, \sigma_{2}$ die Berührungspunkte auf $\varkappa_{s}, \tau_{1}, \tau_{2}$ die auf $\varkappa_{k}$ sind. Die Tangenten schneiden sich auf $O \Pi_{\text {; }}$ es sei $I I$ der Schnittpunkt. $O a, O b$ liegen zu $O \sigma_{1}, O \tau_{1}$ und za $O \sigma_{3}, O \tau_{2}$ harmonisch; ferner sind $O H, O \Pi z \mathrm{zu} O \sigma_{1}, O \sigma_{9}$ und zu $O \tau_{1}, O \tau_{2}$ harmonisch konjugiert. Es sei $k_{0}$ ein Kegelschnitt, der $O a, O b$ in $A^{\prime}, B^{\prime}$ berührt; die Polare $A^{\prime} B^{\prime}$ von $O$ werde mit $o$ bezeichnet. Wir projizieren von $O$ aus die Punkte von $\varkappa_{s}, x_{t}$ auf $k_{0}$ und auf $o$. Ein Punkt $s$ von $\psi_{s}$ wird nach $S^{\prime}$ auf $o$, und nach $S$ und $S^{*}$ auf $k_{0}$ projiziert, wo dann $S, S^{*}$ zu $O$ und $S^{\prime}$ konjugiert sind. In ähnlicher Weise werde die Projektion eines Punktes auf $o$ wie z. B. $I$ mit $I^{\prime}$ bezeichnet.

Eine Gerade $l$ schneidet $\varkappa_{s}$ in $s_{1}, s_{9} ; \varkappa_{t}$ in $t_{1}, t_{z}$. Die Punktpaare $S_{1}^{\prime}, S_{2}^{\prime} ; T_{1}^{\prime}, T_{2}^{\prime} ; A^{\prime}, B^{\prime}$ gehören derselben Involution an. Die Kegelschnitte durch $T_{1}, T_{1}^{*} ; T_{3}, T_{2}^{*}$ schneiden auf $a$ eine Involution aus; ihr gehören die Paare $A^{\prime}, B^{\prime} ; T_{1}^{\prime}, T_{2}^{\prime}$ an. Folglich gibt es einen (und nur einen) Kegelschnitt $k$ durch $T_{1}, T_{1}^{*} ; T_{2}, T_{2}^{*} ; S_{1}^{\prime}, S_{2}^{\prime}$. Lassen wir $l$ variieren, so erhalten wir ein Kegelschnittsystem $k$, von dem wir zeigen wollen, da $B$ alle $k$ durch zwei feste Punkte gehen. Es muß jedoch dabei das Büschel 
ausgenommen werden, dessen Kegelschnitte $k_{0}$ in $A^{\prime}, B^{\prime}$ berühren. Um unsere Behauptung zu beweisen, nehmen wir die Punkte $T_{1}, T_{1}^{*}$ fest an, so dab die Gerade $l$ sich um einen festen Punkt $x$ auf $x_{t}$ dreht. Die Paare $S_{1}^{\prime}, S_{2}^{\prime}$ bilden eine feste Involution, und ein beliebiger von den hier auftretenden Kegelschnitten $k$ ist aus $S_{1}^{\prime}, S_{2}^{\prime}$ und $T_{1}$ bestimmt. Zwei solche Kegelschnitte schneiden sich in $T_{1}, T_{1}^{*} ; Q, Q^{*}$. Weil nun die Kegelschnitte durch diese vier Punkte auf $o$ die eben betrachtete Involution ausschneiden, sind sie mit den Kegelschnitten $k$ identisch. Wenn $l$ durch $O$ geht, zerfällt $k$ in das Geradenpaar $O x, O H$; also müssen $Q, Q^{*}$ auf $O H$ liegen. Lassen wir nun die Gerade $l$ sich um einen anderen festen Punkt $y$ auf $z_{t}$ drehen, so erhalten wir ein zweites Büschel von Kegelschnitten $k$, das auch zwei Grundpunkte auf $O H$ hat. Die beiden Büschel, die zu $x$ und $y$ gehören, haben einen Kegelschnitt, den von $l=x y$ bestimmten, gemein. Also müssen die Punkte $Q, Q^{*}$ fest sein. Sie können auf. folgende Weise erhalten werden: Wenn $l$ mit $\sigma_{1} \tau_{1}$ zugammenfällt, geht $k$ in die beiden Tangenten über, die aus $\Sigma_{1}^{\prime \prime}$ an $k_{0}$ gelegt werden können and die $O H$ in $Q, Q^{*}$ schneiden. Ebenso liefert $l=\sigma_{\mathrm{g}} \tau_{\mathrm{g}}$ die beiden Tangenten an $k_{0}$ von $\Sigma_{2}^{\prime}$ aus; $T_{1} T_{1}^{*} T_{2} T_{2}^{*}$ ist ein in $k_{0}$ eingeschriebenes Viereck, die Tangenten in diesen Punkten an $k_{0}$ bilden ein um $k_{0}$ umschriebenes Vierseit. Die Diagonalen des letzteren sind $O \Pi, O$ und $O H$; sie verbinden die Diagonalpunkte $\Pi^{\prime}, Q^{\prime}, O$ des ersteren und bilden ein Polardreieck von $k_{0}$.

Wir können nun umgekehrt von $O, o, k_{0}$ and $Q$ ausgehen und haben dann den folgenden Satz:

Sei $k_{0}$ ein fester Kegelschnitt, o die Polare eines Punktes $O$ in bezug auf $k_{0}$. Unter $k$ seien die Kegelschnitte verstanden, die durch einen festen Punkt $Q$ gehen und in bezug auf welche o die Polare von $O$ ist. $a, b$ seien zwei beliebige Punkte auf den Tangenten von $O$ aus an $k_{0}, x$ das $B$ Buschel, dessen Kegelschnitte $O Q$ in $O$ berühren und durch $a, b$ gehen. Dann lassen sich die $*$ in Paare $x_{s}, x_{t}$ mit folgender Eigenschaft ordnen: Ein Kegelschnitt $k$ schneidet $o$ in zuei, $k_{0}$ in vier Punkten. Die ersteren werden aus $O$ in $s_{1}, s_{2}$ auf $x_{s}$, die letzteren in $t_{1}, t_{2}$ auf $x_{t}$ projiziert. Dann liegen, für jeden $k$, die vier Punkte $s, t$ in einer Geraden.

Um eine für das folgende zweckmäßige Bestimmung von $x_{z}, x_{t} z u$ erhalten, bemerken wir, da $\beta$ diese Kegelschnitte perspektiv liegen mit $\Pi$ als Zentrum, $a b$ als Achse und $C_{s}, O$ als entsprechenden Punkten, wenn $O_{s}$ den zweiten Schnittpunkt von $O \Pi\left[\right.$ mit $\varkappa_{s}$ bezeichnets Wird also $x_{s}$ beliebig, aber $O Q$ in $O$ berührend gewählt, so ist die Achse $a b$ und $C_{s}$ bestimmt; es genügt folglich $I I$ zu bestimmen. Die harmonischen Polaren durch $Q$ in bezug auf $k_{0}$ schneiden eine Involution auf $o$ aus, deren 
Doppelpunkte $\Sigma_{1}^{\prime}, \Sigma_{2}^{\prime}$ sind. Wird diese Involution aus $O$ auf $x_{s}$ projiziert, so ist $I I$ ihr Zentrum. Weil schon $O I I$ bekannt ist, genügt es ein Punktepaar dieser Involution aufzusuchen.

Der Satz läßt verschiedene Anwendungen zu. Hier wollen wir den folgenden Fall betrachten. Es sei $\pi$ eine Ebene und $P$ ein Punkt in $\pi$, $P Q=q$ eine Gerade außerhalb $\pi$. Ein Kegel $K$ mit der Spitze $P$, mit $\pi$ als Symmetrieebene und $q$ als Erzeugender schneidet $\pi$ in zwei Erzeugenden $s_{1}^{\prime}, s_{2}^{\prime}$ und hat zwei Reihen zu $\pi$ senkrechter Kreisschnitte; die Spurlinien der beiden Kreisschnite durch $P$ in der Ebene $\pi$ seien $t_{1}^{\prime}, t_{2}^{\prime}$. $K$ ist offenbar durch Angabe von $s_{1}^{\prime}, s_{\dot{z}}^{\prime}$ oder durch Angabe von $t_{1}^{\prime}, t_{2}^{\prime}$ bestimmt, und zwar kann eines von diesen Geradenpaaren beliebig gewählt werden. Man erhält in dieser Weise eine gewisse Beziehung zwischen den Geraden $s^{\prime}$ und $t^{\prime}$ durch $P$ und zwar der Art, daß ein Paar $s_{1}^{\prime}, s_{z}^{\prime}$ ein Paar $t_{1}^{\prime}$, $t_{2}^{\prime}$ ein-eindeutig bestimmt. Diese Beziehung hat auf Grund der geometrischen Bedeutung der Geradenpaare $s^{\prime}$ und $t^{\prime}$ gewisse Eigenschaften, die es ermöglichen, ihr eine sehr einfache geometrische Erzeugung zu geben. Um diese zu erhalten, betrachten wir die Schnitte der unendlichfernen Ebene mit $\pi, K, q$, den Geradenpaaren $s^{\prime}, t^{\prime}$ und der Normalen $P O$ von $\pi$. Sie seien mit $o$, bzw. $k, Q, S_{1}^{\prime}, S_{2}^{\prime} ; T_{1}^{\prime}, T_{2}^{\prime}$ und $O$ bezeichnet. $K$ ist offenbar durch $P$ und $k$ bestimmt; die Kegelschnitte $k$ gehen durch $Q$, und $o$ ist die Polare von $O$ in bezug auf alle $k$. $k_{0}$ sei der absolute Kegelschnitt. Die Schnittpunkte von $k$ mit $o$ sind $S_{1}^{\prime}, S_{2}^{\prime}$; die Schnittpunkte von $k$ und $k_{0}$ werden aus $O$ durch $O T_{1}^{\prime}, O T_{2}^{\prime}$ projiziert. Wir können nan die Kegelschnitte $x_{s}, x_{t}$ des oben bewiesenen Satzes einführen. Betrachten wir zwei korrespondierende Geradenpaare $s^{\prime}, t^{\prime}$, so schneiden die zu $\boldsymbol{x}$ senkrechten Ebenen durch diese Geraden $\varkappa_{s}$ bzw. $x_{t}$ in den Punkten $s_{1}, s_{2} ; t_{1}, t_{2}$ einer Geraden. Und die Konstruktionen in der unendlich fernen Ebene können in folgender Weise nach $\pi$ verlegt werden: Wir projizieren $\varkappa_{s}, *_{t}$ von $P$ aus; die projizierenden Kegel berühren sich längs der Erzeugenden $P O$, und die gemeinsame Tangentialebene ist die orthogonalprojizierende Ebene von $q$, deren Spurlinie in $\pi$ mit $q^{\prime}$ bezeichnet sei. Schneiden wir diese Kegel mit einer zu $\pi$ parallelen Ebene, und werden die Schnittkurven auf $\pi$ orthogonal projiziert, so erhalten wir zwei Kegelschnitte $x_{s}^{\prime}, x_{t}^{\prime}$, die $q^{\prime}$ in $P$ berühren; zwei korrespondierende Geradenpare $s^{\prime}, t^{\prime}$ durch $P$ schneiden $x_{s}^{\prime}$ bzw. $x_{t}^{\prime}$ in Punkten einer Geraden. Damit ist die gewünschte Erzeugung der Beziehung zwischen $s^{\prime}$ und $t^{\prime}$ erreicht, and es erübrigt, die Konstruktion von $\varkappa_{s}^{\prime}, x_{t}^{\prime}$ anzugeben. Für die Vereinfachung der Erzeugung ist die Bemerkung von besonderem Werte, daß man für $x_{s}^{\prime}, x_{t}^{\prime}$ Kreise wählen kann. Denn im Satze S. 139 können $a, b$ nach $A^{\prime}, B^{\prime}$ verlegt werden; weil hier $A^{\prime}, B^{\prime}$ die Kreispunkte von $\pi$ sind, werden die Kegel durch $x_{s}, x_{t}$ durch die $z a r$ parallelen Ebenen in Kreise 
geschnitten. Nach den oben gemachten Bemerkungen wählen wir für $x_{s}^{\prime}$ einen beliebigen $\mathrm{K}_{\text {reis, }}$, der $q^{\prime}$ in $P$ berïhrt, und wollen auf der Senkrechten $\pi^{\prime}$ zu $q^{\prime}$ durch $P$ den Ähnlichkeitspunkt $\Pi^{\prime}$ เ von $x_{s}^{\prime}, x_{i}^{\prime}$ aufsuchen. Die zueinander senkrechten Ebenen durch $q$ schneiden $x_{s}^{\prime}$ in Punktpaaren einer Involution mit dem Zentrum $\Pi^{\prime}$. Wir können z. B. das zu $x^{\prime}$ symmetrische Paar aufsuchen. In der Fig. 1 ist $q^{(0)}$ die Umlegung von $q$ um $q^{\prime}$. Ein Kreis mit der Mitte $N$ auf $q^{\prime}$, der $q^{(0)}$ berührt, schneidet die Parallele za $\pi^{\prime}$ durch $N$ in $R$; dann ist $P R$ ein Strahl im gesuchten Paare. Schneidet $P R$ den Kreis $\varkappa_{s}^{\prime}$ in $X$, so ist $X \Pi^{\prime}$ zu $\pi^{\prime}$ senkrecht, also $\Pi^{\prime}$ und mit-

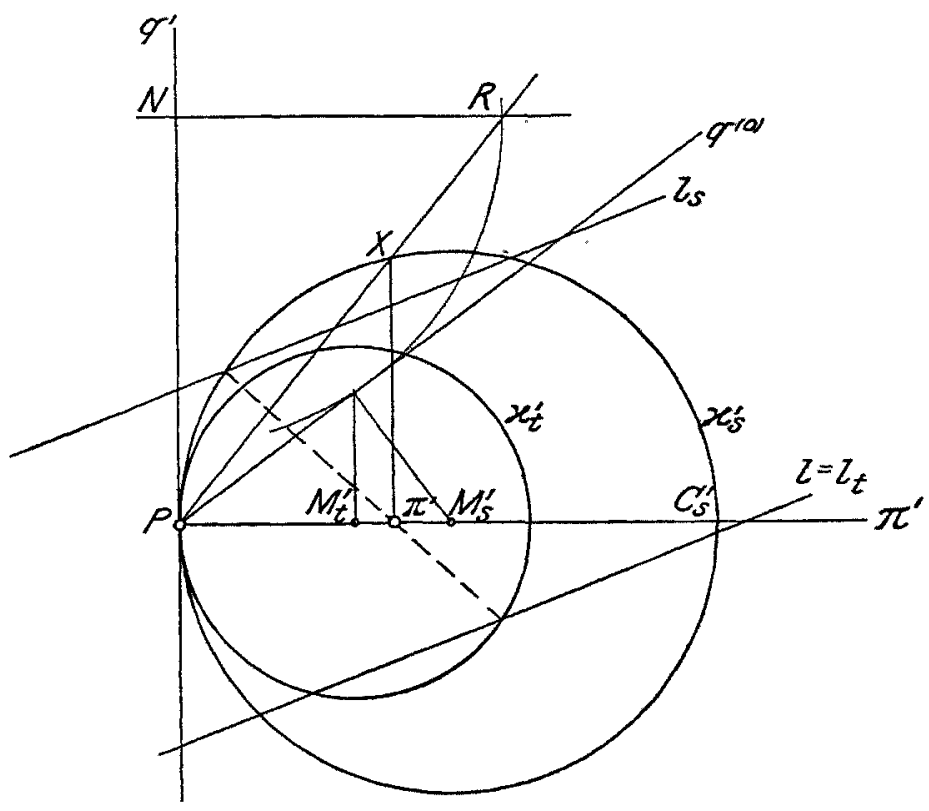

Fig. 1.

hin $\varkappa_{t}$ bestimmt. Wir können aber eine noch einfachere Konstruktion von . «t angeben; dieselbe könnte in Zusammenhang mit dem Beweise des Satzes S. 139 hergeleitet werden, sie ergibt sich auch leicht aus der schon erhaltenen. Es sei $\gamma$ der Winkel zwischen $q$ und $P O, \varphi$ der Winkel, den $P R$ mit $\pi^{\prime}$ bildet. Dann zeigt unsre Figur, daß $\cos \gamma \cdot \operatorname{tg} \varphi=1$. Wenn $C_{s}^{\prime}$ der zweite Schnittpunkt von $\pi^{\prime}$ und $x_{s}^{\prime}$ ist, hat man

und, weil $\Pi^{\prime}$ der Ahnlichkeitspunkt ist,

$$
\left(\Pi^{\prime} X\right)^{2}=P \Pi \cdot \Pi^{\prime} C_{8}^{\prime} \text { oder }\left(\frac{\Pi^{\prime} X}{P \Pi^{\prime}}\right)^{2}=\frac{\Pi^{\prime} C_{8}^{\prime}}{P \Pi^{\prime}}
$$

$$
\frac{\Pi^{\prime} C_{s}^{\prime}}{P \Pi^{\prime}}=\frac{r_{s}}{\gamma_{t}},
$$


wenn $r_{s}, r_{t}$ die Halbmesser sind. Es ist also

Folglich:

$$
\begin{gathered}
\frac{r_{s}}{r_{t}}=\left(\frac{\Pi^{\prime} X}{P \Pi^{\prime}}\right)^{2}=\operatorname{tg}^{2} \varphi=\frac{1}{\cos ^{2} \gamma}, \\
r_{t}=r_{s} \cos ^{2} \gamma .
\end{gathered}
$$

$P$ sei ein Punkt der Ebene $\pi, q=P Q$ eine Gerade, in $\pi$ nicht enthalten und zu $\pi$ nicht normal, $q^{\prime}$ deren Orthogonalprojektion auf $\pi, K$ die Kegel, die $P$ als Spitze, $\pi$ als Symmetrieebene und $q$ als Erzeugende haben. Dann lassen sich die Kreise $x^{\prime}$, die $q^{\prime}$ in $P$ berühren, in Paaren $x_{s}^{\prime}, x_{t}^{\prime}$ so ordnen, daß die Erzeugenden von $K$ in $\pi$ den Kreis $x_{s}^{\prime}$, die Kreisschnitte von $K$ durch $P$ und $z u \pi$ senkrecht den Kreis $x_{t}^{\prime}$ in Punkten schneiden, die in einer Geraden liegen. Und zwar kann der Mittelpunkt $M_{s}^{\prime}$ von $x_{s}^{\prime}$ beliebig gewählt werden; er bestimmt den Mittelpunkt $M_{t}^{\prime}$ von $x_{i}^{\prime}$, indem man das Lot von $M_{s}^{\prime}$ auf die Umlegung $q^{(0)}$ fällt und vom Fußpunkte dieses Lotes ein Lot auf $P M_{s}^{\prime}$ fällt, das dann $M_{t}^{\prime}$ ausschneidet.

Es wäre nun möglich, nachdem dies Resultat gewonnen ist, es nachträglich auf elementar-geometrischem Wege zu verifzieren; was ich jedoch hier nicht ausführe. Ich möchte aber noch die Bemerkung hinzufügen, da $B$ die Beziehung zwischen den Geradenpaaren $s^{\prime}$ und $t^{\prime}$ ein spezieller Fall einer allgemeinen Beziehung zwischen zwei $\infty^{1}$-Systemen von der Art ist, daß jedes Paar von Elementen des einen Systems ein Paar des anderen ein-eindeutig bestimmt. Anf diese allgemeine Beziehung und deren geometrische Eirzeugung werde ich in einer späteren. Note zurückkommen..

Je nachdem die Sekante $l$ den Kreis $x_{t}^{\prime}$ reell schneidet oder nicht, sind die zu $\pi$ senkrechten Kreisschnitte reell oder imaginär. Wenn $l x_{t}^{\prime}$ berührt, erhält man die Umdrehungskegel, die $q$ enthalten und symmetrisch zu $x$ liegen. Für die Mittelpunksflächen zweiten Grades, die $K$ als Asymptotenkegel haben, ergibt sich die Bedeutung der Geradenpaare $s^{\prime}$ und $t^{\prime}$ sofort. Ferner ist der obige Satz für die Konstruktion der Fokalstrahlen eines Kegels verwendbar. Denn wenn der unendlich ferne Kegelschnitt $k$ in bezug auf $k_{0}$ reziproziert wird, geht $K$ in den Normalenkegel $\bar{K}$ über, $t_{1}^{\prime}, t_{2}^{\prime}$ in die Fokalstrahlen $\bar{t}_{1}, \bar{t}_{3}$ und $\bar{s}_{1}, \bar{s}_{2}$ behalten ihre Bedeutung. Die den Kreisen $x_{s}^{\prime}, x_{t}^{\prime}$ entsprechenden Kegelschnitte $\bar{x}_{s}, \bar{x}_{t}$ ergeben sich leicht, wenn man $z$. B. den Kreis mit der Mitte $P$ und dem Radius $\sqrt{-1}$ zum Fundamentalkegelschnitt wählt. Ich fasse nur das Resultat zusammen: $\bar{K}$ sind die Kegel, die $x$ als Symmetrieebene und den Punkt $P$ in $\pi$ als Spitze haben, und die eine gegebene Ebene $\bar{q}$ berühren. Die Erzeugenden eines Kegels $\bar{K}$ in $\pi$ seien $\vec{\beta}_{1}, \vec{s}_{2}$; die Fokalstrahlen desselben Kegels in $\pi$ seien $\bar{t}_{1}, \bar{t}_{\mathrm{g}}$. Dann lassen sich die Parabeln $\bar{x}$, die die Spurlinie $q^{\prime}$ von $\bar{q}$ 
in $x$ als gemeinsame Achse, den Punkt $P$ als gemeinsamen Brennpunkt haben, in Paare $\bar{\varkappa}_{s}, \bar{\varkappa}_{t}$ so ordnen, da $\beta$ die Tangenten an $\bar{x}_{s}$, die $z u \bar{s}_{1}, \bar{s}_{2}$ parallel sind, und die zu $\bar{t}_{1}, \bar{t}_{2}$ parallelen Tangenten an $\bar{x}_{t}$ sich in einem Punkt schneiden. Die Parameter won $\bar{x}_{s}, \bar{x}_{t}$ verhalten sich wie $\cos ^{2} \gamma: 1$, wenn $\gamma$ der Winkel zwischen $\pi$ und $\bar{q}$ ist. Die Umdrehungskegel $\bar{K}$ ergeben sich folglich, wenn der Schnittpunkt der erwähnten Tangenten an $\bar{x}_{s}$ die Parabel $\bar{x}_{t}$ durchläuft.

\section{$\S 2$.}

Von der oben erhaltenen Konstruktion der Geradenpare $s^{\prime}$ und ' $t^{\prime}$ wollen wir eine spezielle Anwendung machen. Es handelt sich um die räumliche Erzeugung der Beziehung zweier affinen ebenen Systeme mittels Parallelprojeltion. Zur Orientierung mögen einige Bemerkungen über die affine Verwandtschaft vorausgeschickt werden.

Zwei ebene projektive Systeme sind affin, wenn die unendlich fernen Geraden sich entsprechen. Liegen die Systeme in derselben Ebene, so ist also die unendlich ferne Gerade dieser Ebene eine Doppelgerade in der projektiven Beziehung. Weil dann schon zwei entsprechende Geraden gegeben sind, wird die Beziehung durch Angabe zweier Dreiecke bestimmt. Bei zwei entsprechenden Geraden entsprechen sich die unendlich fernen Punkte; die Geraden tragen daher ähnliche Punktreihen $A, B, C, \ldots$; $A^{\prime}, B^{\prime}, C^{\prime}, \ldots$ Es muß also $A B: A^{\prime} B^{\prime}$ konstant sein; zu jeder Richtung $a$ des einen Systems gibt es eine Zahl $\nu=\nu(a)$, die das konstante Verhältnis zwischen einer Strecke von dieser Richtung und der entsprechenden Strecke angibt. Ich erinnere ferner daran, daß die Flächeninhalte entsprechender Figuren ein konstantes Verhältnis $\sigma$ haben, was sich übrigens aus den Bemerkungen unten ergeben wird. Die Doppelelemente können bei der Affinität, wie bei der allgemeinen Projektivität, in verschiedener Weise auftréten. Sie können speziell in unendlicher Anzahl vorhanden sein. Es gibt dann eine Gerade $p$, die nur Doppelpunkte trägt, und einen Punkt $O$, der nur Doppelstrahlen trägt. Es kann dabei $O$ auberhalb oder auf $p$ liegen. Die Systeme sind dann perspektiv mit $p$ als Perspektivitätsachse und $O$ als Zentrum. Wenn $p$ endlich, $O$ unendlich ist, liegt die eigentliche perspektive Lage vor; die Beziehung ist dann offenbar von $p$ und irgend zwei entsprechenden Punkten bestimmt. Wird in diesem Falle die Ebene des einen Systems um $p$ um einen beliebigen Winkel gedreht, so kann das eine System aus dem anderen durch Parallelprojektion abgeleitet werden. Ungekehrt gibt die Parallelprojektion eines ebenen Systems und dessen Umlegung in die Bildebene immer affine Systeme in perspektiver Lage. Die Bedingung dafür, daß zwei affine ebene Systeme in perspektive Lage gebracht werden können, ist offenbar die, daß es eine 
reelle Richtung mit $v=1$ gibt. Nun bildet gerade die Affinität den Ausnahmefall bei dem allgemeinen Satz von Poncelet, dab zwei ebene projektive Systeme in reelle perspektive Lage gebracht werden können. Man kann sich dies klarmachen, wenn man z. B. einen Kreis des einen Systems und die entsprechende Ellipse betrachtet. Wird das eine System so verschoben, daß die Mittelpunkte dieser Kurven "sich decken, so müssen sich der Kreis und die Ellipse reell schneiden, wenn eine reelle perspektive Lage möglich ist. Es ergibt sich hieraus, daß eine reelle affine Transformation nicht immer auf eine reelle perspektive Transformation zurückgeführt werden kann (es muß vielmehr eine gewisse Ungleichung erfüllt sein; vgl. z. B. Schröter, Theorie der Obərflächen zweiter Ordnung und der Raumkurven dritter Ordnung usw. (1880), S. 412ff.), daß sie aber immer aus einer Ähnlichkeitstransformation und einer perspektiven Transformation zusammengesetzt werden kann. Daraus läßt sich z. B. das oḅenerwähnte Vorhandensein der Konstante $\sigma$ erschließen.

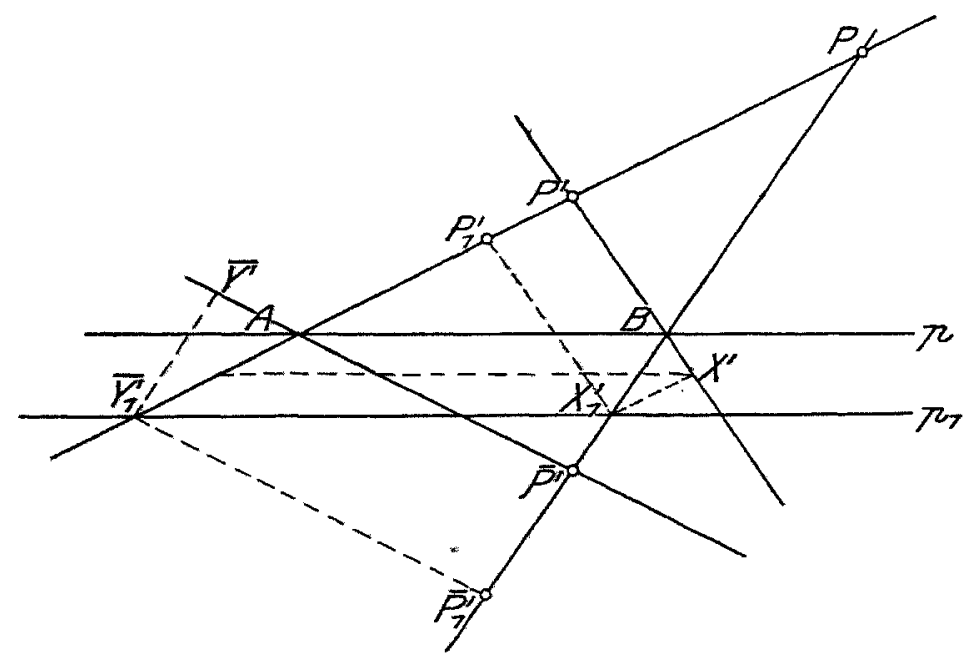

Fig. 2.

Nach dieser Rekapitulation einiger Sätze der elementaren projektiven Geometrie wollen wir eine besondere Definition der perspektiven Affinität herleiten. $p$ sei die Perspektivitätsachse, $P$ und $P^{\prime}$ zwei entsprechende Punkte (Fig. 2). Die Systeme wollen wir mit $(P)$ und $\left(P^{\prime}\right)$ bezeichnen; $(P)$ soll festliegen, $\left(P^{\prime}\right)$ dagegen werde in der Ebene $\pi$ des Systems $(P)$ versehoben and gespiegelt. Bei der Spiegelung von $\left(P^{\prime}\right)$ an $p$ geht $\left(P^{\prime}\right)$ in das symmetrische System $\left(\bar{P}^{\prime}\right)$ über, das mit $(P)$ perspektiv liegt mit $p$ als Achse und dem unendlich fermen Punkt von $P \bar{P}^{\prime}$ als Zentrum. Die Affinitätsrichtungen $P P^{\prime}, P \bar{P}^{\prime}$ seien mit $\varepsilon_{1}, s_{3}$ bezeichnet, wenn sie als 
dem Systeme $(P)$ angehörig betrachtet werden; ihnen entsprechen in $\left(P^{\prime}\right) s_{1}^{\prime}=s_{1}, s_{2}^{\prime}=P^{\prime} B$. Einer zu $p$ parallelen Strecke in $(P)$ entspricht eine gleich lange in $\left(P^{\prime}\right)$ und $\left(\bar{P}^{\prime}\right)$. Wird daher $\left(P^{\prime}\right)$ in der Richtung $s_{1}$ oder $\left(\bar{P}^{\prime}\right)$ in der Richtung $s_{2}$ verschoben, so wird die perspektive Lage beibehalten, und die Affinitätsrichtungen werden nicht geändert. Bei der Verschiebung liegt immer ein Punkt $X^{\prime}$ auf $P^{\prime} B$ mit seinem entsprechenden auf $P B$, bzw. ein Punkt $\bar{Y}^{\prime}$ auf $\bar{P}^{\prime} A$ mit dern entsprechenden auf $P A$ vereinigt. Es sei (Fig. 3) $C$ der Schnittpunkt der Mittennormale von $P P^{\prime}$ mit $p$; dann ist $P C=P^{\prime} C=\bar{P}^{\prime} C$. $P C$ kann daher als Perspektivitätsachse gewählt werden und, um die Systeme in perspektive Lage mit $P C$ als Achse zu bringen, hat man $\left(P^{\prime}\right)$ bzw. $\left(\bar{P}^{\prime}\right)$ um $C$ zu drehen. Weil nun $A B^{\prime} P^{\prime} C=A B^{\prime} \bar{P}^{\prime} C=A B P C$, so gelangt $B^{\prime}$, bei der Drehung von $\left(P^{\prime}\right)$, nach $B_{1}^{\prime}$ auf $P B$. Die Affinitäts-

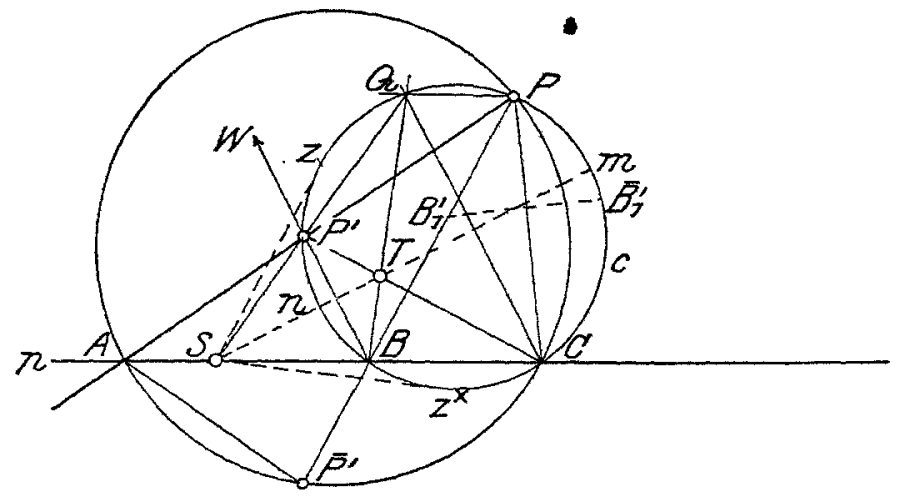

Fig. 3.

richtung in der neuen perspektiven Lage von $(P)$ und $\left(P^{\prime}\right)$ ist mithin $s_{\mathbf{x}^{\prime}}$. Bei der Drehung von $\left(\bar{P}^{\prime}\right)$ gelangt $\bar{B}^{\prime}$ nach $\bar{B}_{1}^{\prime}$, dem zu $B_{1}^{\prime}$ symmetrischen Punkt bezüglich $P C$. Nun liegen die Punkte $P, P^{\prime}, B, C$ auf einem Kreise $c$; der Symmetrie halber geht $c$ durch $\bar{B}_{1}^{\prime}$. Die Affinitätsrichtung $B \bar{B}_{1}^{\prime}$ ist folglich $s_{1}$. Die Richtungen $A B$ und $P C$, die Strecken tragen, denen gleich lange entsprechen, nennen wir Affinitätsachsen; wir wollen sie mit $r_{1}, r_{2}$ bezeichnen. Es kann deren drei nicht geben, es sei denn, $\mathrm{da} B$ die Systeme kongruent (symmetrisch) sind. Wird in der Fig. $3 P Q$ gezogen, so ist $A Q P P^{\prime}=A B P C^{\prime}$; übrigens liegen die Punkte $A, C, P, \bar{P}^{\prime}$ auf einem Kreise. Es gibt zwei Affinitätsachsen und zwei zugehörige Affinitätsrichtungen, von den Verschiebungen abgesehen also vier perspektive Lagen von $(P)$ und $\left(P^{\prime}\right),\left(\bar{P}^{\prime}\right)$. Die Achsen und die Affinitätsrichtungen haben dieselben Bissektrizen.

Gehen wir umgekehrt von zwei Paaren von Richtungen $s_{1}, s_{3} ; r_{1}, r_{2}$ aus, die dieselben Bissektrizen haben, so können $s_{1}, s_{2}$ als die Affinitäts- 
richtungen, $r_{1}, r_{\exists}$ als die Affinitätsachsen einer perspektiven Affinität betrachtet werden, die dann eindeutig bestimmt ist. Denn in perspektiver Lage mit $p \| r_{1}$ als Perspektivitätsachse entspricht z. B. $s_{1}$ sich selbst. Ist $P$ beliebig und wird $P A \| s_{1}, A \bar{P}^{\prime}$ symmetrisch zu $A P$ bezüglich $p$ und $P \bar{P}^{\prime} \| s_{\mathrm{a}}$ gezogen, so ist $\bar{P}^{\prime}$ and mithin $P^{\prime}$ bestimmt. Eine perspektive Affinität kann durch Angabe der beiden Affinitätsachsen in dem einen System und der beiden Affinitätsrichtungen definiert werden. Für die Achsen ist die oben eingeführte Verhältniszahl $\nu=1$; für die Affinitätsriehtungen ist $v=\sigma$, was sich z. B. aus der Betrachtung eines Parallelogramms in $(P)$ ergibt, dessen Seiten die Richtungen $r_{1}, s_{1}$ haben.

Bei den vorhergehenden Betrachtungen haben wir die Punkte $A$ und $C$ endlich angenommen. Sie können unendlich fern werden, und man erhält dann spezielle Affinititen, inflem sich die vier Richtungen $s$ und $r$ auf zwei reduzieren, was in dem oben betrachteten Fall nicht möglich ist. Wenn $A$ unendlich fern ist, fallen $s_{1}$ und $r_{1}, s_{9}$ und $r_{9}$ zusammen; $\sigma$ ist dann und offenbar nur dann $=1$ (flächentreue Affinität). Wenn $C$ unendlich fern ist, muß $s_{1}$ anf $r_{1}$ senkrecht stehen; dann fallen $s_{1}$ und $s_{3}, r_{1}$ und $r_{a}$ zusammen (orthogonale Affinität).

Indem wir jetzt zur perspektiven Lage von $(P)$ und $\left(P^{\prime}\right)$ mit der Perspektivitätsachse $p \mid r_{1}$ zurückkehren, drehen wir die Ebene $\pi^{\prime}$ von $\left(P^{\prime}\right)$ um einen beliebigen. Winkel um $p . \quad\left(P^{\prime}\right)$ wird dann aus $(P)$ durch Parallelprojektion in der Richtung $P P^{\prime}=g$ oder auch $(P)$ aus $\left(P^{\prime}\right)$ erzeugt. Es sei $A B C \ldots$ ein Polygon in $(P)$; die zu $g$ parallelen Strahlen durch seine Ecken bestimmen ein Prisma $\mathfrak{F}$, aus dem das entsprechende Polygon $A^{\prime} B^{\prime} C^{\prime} \ldots$ von $\pi^{\prime}$ ausgeschnitten wird. Zunächst suchen wir auf $\mathfrak{B}$ alle ebenen Schnitte auf, die mit $A^{\prime} B^{\prime} C^{\prime} \ldots$ in der Weise kongruent sind, dab die bei der Kongruenz entsprechenden Ecken auf denselben Kantenlinien liegen. Jedes derartige Polygon und nur ein solches bestimmt mit $A B C \ldots$ diejenige Affinitat zwischen $(P)$ und $\left(P^{\prime}\right)$, von der wir ausgegangen sind, und die durch die Paare $s, r$ definiert ist. Die mit $\pi^{\prime}$ parallelen Ebenen liefern solche Schnitte und schneiden $\pi$ in Parallelen zu $r_{1}$. Wir erhalten ferner solche Schnitte durch die Ebenen, die $z u \pi^{\prime}$ in bezug auf eine zu $g$ senkrechte Ebene symmetrisch sind. Diese müssen dann $\pi$ in Parallelen zu $r_{\sharp}$ schneiden. Die verschiedenen perspektiven Lagen in $\pi$ ron $(P)$ and $\left(P^{\prime}\right),\left(\bar{P}^{\prime}\right)$, die-wir oben betrachteten, entsprechen also den Parallelverschiebungen von $\pi^{\prime}$ in der Richtung $g$ und den Spiegelungen von $x^{\prime}$ an zu $g$ senkrechten Ebenen. Es kann nun aber $g$ und mithin $\$$ variiert werden. Bei der Drehung von $\pi^{\prime}$ um $p$ erzeugt, $g$ einen Kegel $K$, der $P$ als Spitze und den Kreis über dem Durehmesser $P^{\prime} \bar{P}^{\prime}$ als Basiskurve hat. 'Man erhält dann $\infty^{1}$ Prismen $\mathfrak{P}$, 
deren Kantenlinien zu den Erzeugenden von $K$ parallel sind. Die ebenen Schnitte $A_{1} B_{1} C_{1} \ldots, A_{2} B_{2} C_{2} \ldots$ irgend zweier von diesen Prismen sind vermittels $A B C \ldots$ affin, und die entsprechenden Punkte $A_{1}, A_{2}$ liegen auf Kantenlinien durch $A$. Nun können, wie unten gezeigt wird, diese Schnitte in unendlich vielen Weisen so gewählt werden, daß die genannte Affinität in Kongruenz übergeht. Unter diesen kongruenten Schnitten findet sich der oben mit $A^{\prime} B^{\prime} C^{\prime} \ldots$ bezeichnete, und dieser ist der einzige, der für alle Prismen gemeinsam ist; dabei sind immer die mit $A B C \ldots$ kongruenten Schnitte außer Betracht gelassen worden. Der Kegel $K$ definiert daher die Affinität zwischen $(P)$ und $\left(P^{\prime}\right)$ in der Weise, daß $\left(P^{\prime}\right)$ aus dem für alle $\mathfrak{P}$ gemeinsamen Schnitt bestimmt ist. Dies ergibt sich auch sofort, wenn wir die Beziehung von $K$ zu den Paaren $r, s$ näher betrachten. $\pi$ ist eine Symmetrieebene von $K ; s_{1}, s_{2}$ sind die Erzeugenden in $\pi$. Der Basiskreis über $P^{\prime} \bar{P}^{\prime}$ steht auf $p_{1}$ senkrecht. Nun sind die Kreisschnitte von $K$ bekanntlich Wechselschnitte zu den Erzeugenden in $\pi$, und weil $r_{1}, r_{2}$ dieselbe Eigenschaft haben, muß der zweite Kreisschnitt auf $r_{2}$ senkrecht stehen. Jeder Kegel zweiter Ordnung $K$ definiert also eine reelle perspektive Affinität. Wird dieselbe in die zu den reellen Kreisschnitten senkrechte Symmetrieebene $\pi$ verlegt, so liefern die Erzeugenden in $\pi$ die Affinitätsrichtungen, die Normalen zu den Kreisschnitten die Affinitätsachsen und die Erzeugenden von $K$ die Richtungen, in denen das eine System in dem anderen parallelprojiziert werden kann. Es muß jedenfalls vorausgesetzt werden, da $K$ eigentlich und auch kein Umdrehungskegel ist. Im letzteren Falle artet die Affinität aus; bei einer orthogonalen Affinität geht $K$ in eine zu $\pi$ senkrechte (Doppel-) Ebene über.

Die Anwendung des Satzes S. 139 ist nun unmittelbar. Wir betrachten das System $(P)$ in $\pi$ und eine nicht $z \mathrm{u} \pi$ senkrechte Richtung $P Q=q$. Die Aufgabe, die mit $(P)$ affinen Systeme $\left(P^{\prime}\right)$ zu bestimmen, die in $(P)$ in der Richtung $q$ parallelprojiziert werden können, erhält die folgende Lösung. Nach $\S 1$ werden die Kreise $x_{s}, x_{t}$ (wir lassen hier die Akzente weg) konstruiert; jeder Sekante $l$ entspricht dann eine bestimmte Affinität der verlangten Eigenschaft, indem ihre Schnittpunkte mit $x_{s}$ bzw. $x_{t}$ von $P$ aus projiziert die Affinitätsrichtungen bzw. die Normalen der Affinitätsachsen liefern. Die letzteren sind reell, wenn $l$ den Kreis $\varkappa_{t}$ reell schneidet; geht $l$ durch $P$, so erhält man eine unbestimmte orthogonale Affinität mit der Achse senkrecht zu $q^{\prime}$. Die angegebene Konstruktion liefert in erster Linie die Normalen der Achsen. $l$ schneide $\psi_{s}$ in $s_{1}, s_{2}$, so daß $P s_{1}=s_{1}, P s_{2}=s_{2} . \quad I$ ist der Ähnlichkeitspunkt zweier Systeme, die wir mit $\left(x_{s}\right)$ und $\left(x_{t}\right)$ bezeichnen können. Wird l. als zum Systeme $\left(x_{t}\right)$ gehörig betrachtet, $l=l_{t}$, so schneidet die 
entsprechende Gerade $l_{\alpha}$ den Kreis $x_{s}$ in $r_{1}, r_{2}$, so daß $P r_{1}=r_{1}, P r_{2}=r_{3}$ die Achsen sind. Denn $P r_{\mathrm{Z}}$ in $\left(x_{s}\right)$ entspricht $C_{t} t_{1}$ in $\left(\varkappa_{t}\right)$, wenn $t_{1}, t_{3}$ die Schnittpunkte von $l$ and $\varkappa_{t}$ sind, und diese Geraden sind parallel. Es ist also $P r_{g} \perp P t_{g}$, wie die Behauptung besagt. Dies liefert sofort die Antwort auf die Frage, wenn $s_{1}, s_{2}$ mit $r_{1}$ bzw. $r_{2}$ zusammenfallen. Die flächentreuen Affinitäten werden also von den Sekanten $l$ durch den Ahnlichkeitspunkt $I$ geliefert $\left.{ }^{1}\right)$. Wir können uns die allgemeinere Aufgabe stellen, die Affinitäten mit gegebenem Flächenverhältnis $\sigma \mathrm{zu}$ bestimmen. Aus der Fig. 3 ergibt sich leicht

$$
\sigma=\sin c: \sin \beta,
$$

wenn $\alpha$ der Winkel $\left(r_{1}, r_{2}\right)$ oder $\left(t_{1}, t_{2}\right)$ and $\beta$ der Winkel $\left(s_{1}, s_{z}\right)$ ist. Es müssen also die Sehnen von $l$ in $x_{s}$ und $x_{i}$ ein konstantes Verhältnis haben und mithin $l$ die gemeinsame Tangente an zwei mit $x_{s}$ bzw. $x_{t}$ konzentrischen Kreisen sein. Diese Kreise werden einander ein-eindeutig zugeordnet, wie sofort klar ist. Wir wollen die Einhüllende $E$ von $l$ bestimmen und beweisen zu diesem $\mathrm{Zwecke}$ zunächst den Hilfssatz:

Es seien $c_{1}, c_{2}$ zwei Büschel Kegelschnitte mit zwei gemeinsamen Grundpunkten $a, b$. Die Kegelschnitte $c_{1}$ haben in $a, b$ feste Tangenten, ebenso die $c_{2}$. Ordnen wir die Kegelschnitte der beiden Büschel einander projektiv zu, so umhüllen die gemeinsamen Tangenten entsprechender Kegelschnitte eine Kurve viester Klasse. Wenn in den beiden tangentiellen Büscheln der Kegelschnitt $a, b$-sich selbst entspricht, so zerfällt die Einhüllende' in zuci Kegelschnitte $a, b$ und $E$. . Denn die Tangenten der Einhüllenden von einem Punkte $U$ aus sind die gemeinsamen Strahlen zugeordneter Pare in den beiden aufeinander projektiv bezogenen Involutionen von Tangenten aus $U$ an den $c_{1}$ und $\operatorname{den} c_{2}$. Es gibt deren vier. Wenn der Kegelsehnitt $a, b$ sich selbst entspricht, gehören $U a, U b$ für jede Lage von $U$ zu diesen Tangenten. Der Kegelschnitt $a, b$ bildet also einen Teil der Einhüllenden; der Rest ist der Kegelschnitt $E$.

Wir wählen nun für $a, b$ die Kreispunkte und als Tangenten von $c_{1}, c_{2}$ in diesen Punkten die nach ihnen gehenden Strahlen von $M_{s}$ bzw. $M_{t}$ aus. Dann stellen $c_{1}, c_{\mathrm{g}}$ die Büschel der $z \mathbf{z} \varkappa_{s b} \varkappa_{t}$ konzentrischen Kreise dar, die, wenn $\sigma$ gegeben ist, projektiv aufeinander bezogen sind und zwar so, daß der Kegelschnitt $a, b$ sich selbst entspricht. Die Gerade $l$ umbüllt folglich einen Kegelschnitt $E$. Unabhängig von dem Werte von $\sigma$ entsprechen sich immer $c_{1}=\varkappa_{s}, c_{2}=\varkappa_{t}$. E gehört also dem von $\varkappa_{s}$ und $\varkappa_{t}$ definierten tangentiellen Büschel an. Demnach:

1) Die Sekanten $l$ durch $P$ können auBer Betracht gelassen werden. 
Die Affinitäten mit gegebenem Flächenverhältnis $\sigma$, die zur gegebenen Projektionsrichtung $q$ gehören, werden von den Tangenten $l$ eines bestimmten, dem tangentiellen Büschel $\varkappa_{s}, \varkappa_{t}$ angehörenden Kegelschnittes $E$ bestimmt. Die konstruktive Behandlung von $E$ ergibt sich unmittelbar hieraus, indem $E$ mit $x_{s}$ perspektiv liegt mit dem Zentrum $I$ und mit $P, C_{s}$ als entsprechenden Punkten. Wird also, für gegebenes $\sigma$, eine einzige der Geraden $l$ konstruiert, was nach (1) leicht auszuführen ist, so ist $E$ bestimmt, und man erhält sofort die Perspektivitätsachse. Es bietet auch keine Schwierigkeiten, die metrischen Verhältnisse bei $E$ festzustellen. $M_{s} M_{t}$ ist eine Achse und $P$ ein Scheitel. Ist $A$ der zweite Scheitel, so liefert (1) sogleich die Länge der Achse $P A$ in $\sigma$ ausgedrückt. Die Fluchtgerade $\omega_{s}$ in $\left(x_{s}\right)$ wird leicht erhalten, ferner ergeben sich die Asymptoten, wenn $E$ Hyperbel, oder die zu $q^{\prime}$ parallele Achse, wenn $E$ Ellipse ist. Man sieht, da $B$ die Tangenten $l$ von $E$ den $\mathrm{Kreis}{ }_{t}$ reell schneiden und folglich reelle Affinitäten liefern, wenn $A$ innerhalb der Strecke $P C_{t}$ fällt. Dabei entsprechen $\operatorname{den} A$ auf $\Pi C_{t}$ Ellipsen $E$, die innerhalb $\varkappa_{t}$ liegen, und den $A$ auf $P I$ Hyperbeln $B$. Ubrigens ist es von vornherein klar, daß bei den reellen Affinitäten

$$
0<\sigma^{2} \leqq 1: \cos ^{2} \gamma=r_{s}: r_{t}
$$

sein muß. Die Anwendung von (1) zeigt, daß wenn $A$ sich $P$ nähert, $\sigma^{2}$ zum Grenzwerte $r_{s}: r_{t}$ strebt. Für diesen Wert von $\sigma^{2}$ geht dann $E$ in das Punktpaar auf $q^{\prime}$ über, das dem tangentiellen Büschel $\varkappa_{s}, x_{t}$ angehört; für $\sigma=1$ geht $E$, wie schon oben gefunden wurde, in das Punktpaar $P, \Pi$ und für $\sigma=0$ in den Kreis $x_{t}$ über. Wenn also $A$ die Strecke $C_{t} P$ beschreibt, geht $\sigma^{2}$ "wachsend von 0 bis $r_{s}: r_{t}$.

Wir geben jetzt zwei Richtungen $q_{1}, q_{2}$ an und wollen die mit $(P)$ affinen Systeme $\left(P^{\prime}\right)$ bestimmen, die sowohl in der Richtung $q_{1}$ wie auch in der Richtung $q_{2}$ in $(P)$ parallelprojiziert werden können. Indem man zu den Betrachtungen des $\$ 1$ zurückkehrt, sieht man, daß die Kegelschnitte $k$ ein Büschel bilden und daB sich also die Sekante $l$ um einen festen Punkt $Y$ dreht. Die Konstruktion ist in der Fig. 4 ausgeführt. $q_{1}, q_{\mathrm{a}}$ werden mit einer zu $\pi$ senkrechten Ebene geschnitten und die Schnittpunkte um die Spurlinie $m$ in $\pi$ umgelegt. Es ist $m \perp q_{1}^{\prime}$ gewählt. Die Umlegungen seien $Q_{1}^{(0)}, Q_{2}^{(0)}$, und $\bar{Q}_{1}^{(0)}$ sei zu $Q_{1}^{(0)}$ symmetrisch bezïglich $m$. Die Verbindungsgeraden $Q_{1}^{(0)} Q_{z}^{(0)}, \bar{Q}_{1}^{(0)} Q_{2}^{(0)}$ schneiden $m$ in Punkten, die aus $P$ durch die Doppelstrahlen der Involution $s$ projiziert werden. Wenn also die Kreise $x_{s}, x_{t}$ in bezug auf $q_{1}$ konstruiert werden, so 'ergibt sich $Y$ sofort aus den Doppelstrahlen der Involution $s$; offenbar mu $\beta Y$ auf $q_{2}^{\prime}$ liegen. Betrachten wir zwei Prismen $\Re_{1}, \Re_{2}$ mit gemeinsamer Basis (deren Kantenlinien zu $q_{1}$ bzw. $q_{2}$ parallel sind), so liefert 
die angegebene Konstruktion die Lösung der Aufgabe, alle ebene Schnitte von $\Re_{1}, \Re_{2} z u$ bestimmen, die in der Weise kongruent sind, daß entsprechende Ecken auf Kantenlinien durch dieselbe Ecke des Basispolygons liegen, Es gibt $\alpha^{1}$ derartige Schnitte; ihre Ebenen gehen zu je zweien durch dieselben Geraden in $\pi$, die Parallelen zu $r_{1}, r_{2}$.

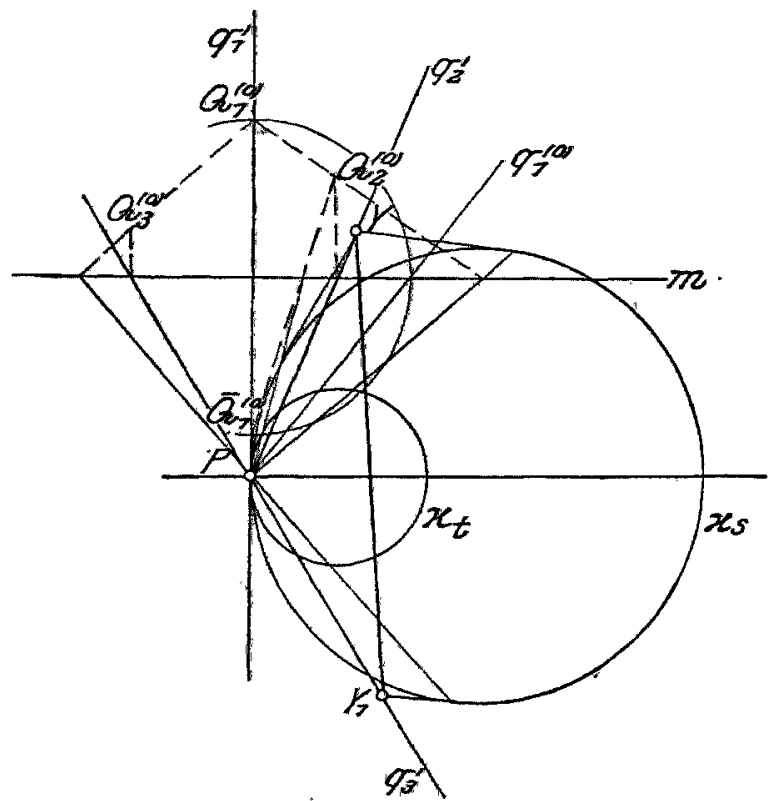

Fig. 4.

Geben wir drei Riehtungen $q_{1}, q_{2}, q_{3}$ an, so ist $K$ vollkommen bestimmt. Werden $\varkappa_{s}, x_{t}$ in bezug auf $q_{1}$ konstruiert und dann, wie oben, das zu $q_{1}, q_{2}$ gehörige Zentrum $Y$, und in entsprechender Weise das zu $q_{1}, q_{3}$ gehörige Zentrum $Y_{1}$ (Fig. 4), so bestimmt $l=Y Y_{1}$ das mit $(P)$ affine System $\left(P^{\prime}\right)$, das in den drei Richtungen $q$ in $(P)$ parallelprojiziert werden kann. Betrachten wir drei Prismen auf gemeinsamer Basis (deren Kantenlinien die Richtungen $q_{i}$ haben), so gibt es ein Polygon und nur eines (die Basis ausgenommen), das auf allen Prismen in der Weise ausgeschnitten verden kann, dab je zwei Ecken, die bei der Kongruenz einander entsprechen, auf Kantenlinien durch denselben Punkt der Basis liegen. Dies Polygon ist reell, wenn $l=Y Y_{1}$ den Kreis $x_{t}$ reell schneidet. 


\section{$\S 3$.}

Die oben hergeleitete Definition der Affinität aus den Affinitätsachsen und den Affinitätsrichtungen ist in einer allgemeineren enthalten. Zunäohst kann die Affinität durch Angabe zweier Paare endlicher Punkte $P, P^{\prime \prime} ; A, A^{\prime}$ und der Reihen auf der unendlich fernen Geraden definiert werden, also durch die Strahlenbüschel in $P$ and $P^{\prime}$ und die endlichen Punkte $A, A^{\prime}$. Indem Verschiebungen und Spiegelungen von $\left(P^{\prime}\right)$ immer einbegriffen sind, kann die Strecke $P^{\prime} A^{\prime}$ aus $P A$ durch Angabe von $\nu(a), a=P A$, bestimmt werden. Ferner kann die Beziehung der zwei Büschel in einer speziellen Weise definiert werden. Wenn $\Lambda(a, b)$ den einen der beiden Winkel $<\pi$ bedeutet, die $a$ und $b$ einschließen, so können immer, bei zwei projektiven Büscheln and dem beliebigen Strahl $a$ des einen, zwei andere derart gefunden werden, daß

$$
\Lambda(a, b)=\Lambda\left(a^{\prime}, b^{\prime}\right) ; \Lambda(a, c)=\Lambda\left(a^{\prime}, c^{\prime}\right) .
$$

(Chasles, Traité de géométrie supérieure (1852), Art. 147). Gibt man im Büschel in $P$ drei Strahlen $a, b, c$ an und fügt man die Bedingung (2) hinzu, so ist das Büschel in $P^{\prime}$ bis auf Verschiebungen und Spiegelungen eindentig bestimmt $\left.{ }^{2}\right)$. Hieraus ergibt sich, daB eine Affinität durch Angabe in $(P)$ dreier Strahlen $a, b, c$ und der Zahl $v(a)$ unter Znfügung der Bedingung (2) bestimmt ist. Für $\nu(a)=1$ geht diese Definition in die oben hergeleitete über. Denn es ist

$$
\Lambda\left(r_{1}, s_{1}\right)=\Lambda\left(r_{1}^{\prime}, s_{1}^{\prime}\right) ; \quad \Lambda\left(r_{1}, s_{2}\right)=\Lambda\left(r_{1}^{\prime}, s_{9}^{\prime}\right)
$$

Setzt man, bei zwei projektiven Strahlenbüscheln mit gemeinsamem Scheitel, die Existenz dreier Strahlen $a, b, c$ mit der Eigenschaft (2) voraus, so ergibt sich sofort, daß die Büschel durch eine Drehung bzu. Spiegelung und Drehung in zwei Weisen in involutorische Lage gebracht werden können. Die Büschel seien $\mathfrak{B}$ und $\mathfrak{B}^{\prime}$; gleichzeitig werde das symmetrische Büschel $\overline{B^{\prime}}$ betrachtet, so daß wir annehmen können, $\mathrm{da}$ der Drehungssinn in $\mathfrak{B}$ und $\mathfrak{B}^{\prime}$ derselbe ist. Ist z. B. der spitze Winkel $(a, b)<\operatorname{der}$ spitze Winkel $(a, c)$, so führt eine Drehung von $\mathfrak{D}^{\prime}$ gleich-

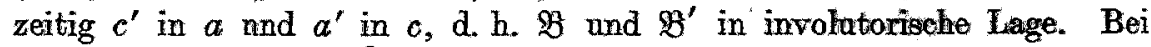
$\overline{9 B}^{\prime}$ führt die Drehring 'von $b^{\prime}$ in $a$ zur involutorisehen Lage. Demnach gilt (2) für jeden Strahl $a$, und ferner bilden die Strahlen $(a, c),(a, b)$ zwei Involutionen ${ }^{i}$ ), die sich am einfachsten ergeben, indem man die Büschel perspektiv macht, wie dies in Fig. 3 schon der Fall ist. Ferner

) Dabeî lassen wir beiseite den Fall, daß die Geradenbüschel kongruent sind.

3) VgI. z. B. Stu rm, Die Lehre von den geometrizehen Vexwandtschaften, I, S. 71. 
können hier, wie bei der allgemeinen Affinität, die entspreehenden Halbstrahlen und mithin die entsprechenden Winkel $<\pi$ unterschieden werden. Die Kreise drurch $P, P^{\prime}$ schneiden die Achse $p$ in Paaren einer Involution, die, aus $P^{\prime}$ projiziert, die Strahleninvolution $\&$ der gleichen entsprechenden Winkel liefert. In ähnlicher Weise schneiden die Kreise durch $P, \bar{P}^{\prime}$ eine Involution auf $p$ ans, die aus $P$ durch die Strahleninvolution $T$ der supplementären entsprechenden Winkel projiziert wird. Die Zentren dieser Involutionen ergeben sich sofort, wenn sie am Kreise $c$ dargestellt werden; es ist $S=P^{\prime} Q \cdot p, T=P^{\prime} C \cdot B Q$. Der dritte Diagonalpunkt des Vierecks $B C Q P^{\prime}$ ist der unendlichferme Punkt $W$ von $P^{\prime} B$; dieser Punkt ist das Zentrum einer Involution $W$ auf $c$, deren Bedeutung wir jetzt feststellen wollen. Oben wurde die Zahl $v$ eingeführt, die das Verhältnis eimer Streake in $(P)$ zur entsprechenden in $\left(P^{\prime}\right)$ angibt. Wollen wir dhe Strahlen $P X$ aufsuchen, denen ein gegebenes $v$ entspricht, so soll $P X: P^{\prime} X=v$ sein, wenn $X$ der Schnittpunkt mit $p$ ist. Es gibt zwei Punkte $X$, die Schnittpunkte von $p$ und einem harmonischen Kreise zu $P, P^{\prime}$ sind. Die harmonischen Kreise zu $P, P^{\prime}$ bilden ein Büschel, die Strahlen mit gleichen $v$ also eine Involution, von der wir schon zwei Paare kennen: $P P^{\prime}, P B$ mit $v=\sigma ; P Q, P C$ mit $y=1$. Die Involution $W$ ist folglich mit der Involution der Strahlen mit gleichen $v$ identisch. Die gegenseitige Beziehung der Involutionen $S, T, W$ ergibt sich übrigens daraus, dab die harmonischen Kreise za $P, P^{\prime}$ das Orthogonalbüschel zu demjenigen mit den Grundpunkten $P, P^{\prime}$ bilden. Sind $M, M^{*}$ die Punkte, in denen zwei zu $P, P^{\prime}$ harmonische Kreise $p$ berühren, so gehört der Kreis mit dem Durchmesser $M M^{*}$ dem Büschel durch $P, P^{\prime}$ an und muß auch durch $\bar{P}^{\prime}$ gehen. Die Doppelstrahlen derjenigen Involution, deren Paare aus den Strahlen mit gleichen $v$ gebildet sind, bilden also einen rechten Winkel, der za $S$ und $T$ gehört; sie ist folglich mit $W$ identisch. Von den oben betrachteten Richtungen $s, r$ gehört das Paar $r_{1}, s_{1}$ zu $S$, das Parr $r_{1}, s_{9}$ zu $T$. Allgemeiner betrachten wir die Strahlen $a, a_{1}$ mit gleichen $v$; die Strahlen $a, a^{*}$ mit den Verhältniszahlen $v$ und $\nu^{*}=\sigma: \nu$. Ist $a$ beliebig, so gehören von den Paren $a, a^{*} ; a, a_{1}^{*}$ das eine der Involution $\$$, das andere der Involution $T$ an, so da $B$ in (2) die Strahlen $b, c$ das Paar in $W$ mit $x=\sigma: v(a)$ bilden. Denn ein Dreiek in $(P)$, von dem zwei Seiten zu $a, a^{*}$ bzw. $a, a_{1}^{*}$ parallel sind, verhält sich zum entsprechenden wie $\sigma=v ; y^{*}$; es muß also der eingeschlossene Winkel bei der Transformation beibehalten oder in den supplementären übergeführt werden.

Mit diesen Bemerkungen sind wohl die Verhältnisse klargelegt, die der oben besprochenen Definition der Affinität zugrunde liegen. In Zusammenhang mit den angegebenen Darstellungen der Involutionen $S, T, W$ 
können noch folgende Kanstrultionen erwähnt werden. Die Involutionen werden bei einer Ahnlichkeitstransformation nicht geändert und Fig. 3 gilt demnach für die allgemeine Affinitat (und für irgend zwei projektive Strahlenbüschel). Durch die Angabe in $(P)$ von $S$ sind daher alle Systeme $\left(P^{\prime}\right)$ definiert, die mit $(P)$ affin und mit einem gewissen System $\left(P^{\prime}\right)$ ahnlich sind. Um ein bestimmtes von diesen Systemen zu exhalten, hat man nur einem Paare in $W$ einen Wert $y$ beizulegen. Z. B. kann der Wert von $\sigma$ angegeben werden oder, was auf dasselbe hinauskommt, der Wert $\sqrt{\sigma}$ für die beiden Doppelstrahlen in $S$. Dabei ist $z$ u bemerken, daB diese Doppelstrahlen immer reell sind, daß ihnen immer der Wert $\sqrt{\sigma}$ entspricht, wie auch $\left(P^{\prime}\right)$ ähnlich transformiert wird, dab aber die Strahien mit $v=\sigma$ reell oder imaginär seín können. Die Strahlen mit vorgegebenem $y$ können auf folgende Weise erhalten werden: Die Betrachtung eines Dreieckes in $(P)$, von dem zwei Seiten zu $a, a_{1}$ parallel sind, and des entsprechenden in $\left(P^{\prime}\right)$ liefert.

$$
\sigma=\nu^{2} \sin c: \sin \beta,
$$

wenn $a=\Lambda\left(a, a_{1}\right), \beta=\Lambda\left(a^{*}, a_{1}^{*}\right)$; für $v=1$ geht diese Gleiehnng in (1) über. Werden die Bezeichnungen so gewählt, daß die Pankte $a, a^{*}$,auf $c$ mit $S$ in einer Geraden liegen, so ist

$$
\sigma: \nu^{2}=\sin c: \sin \beta=S a: S a^{*}=(S a)^{2}:(B z)^{2},
$$

wenn $z$ der eine Doppelstrahl in $S$ und $S z$ also die Tangente an $c$ ist. Es gilt also

$$
\nu(a) \cdot S a=\text { const }=\delta ;
$$

$\delta$ bestimmt mit $S$ die Affinität; aus $S a=\delta: \nu(a)$ werden die beiden Strahlen mit der Verhältniszahl $v(a)$ sofort konstruiert. Damit die Affinitätsachsen reell seien, $m u B S n \leqq \delta \leqq S m$ sein.

Um ein bestimmtes Paar in $S$ oder $T$, d. h. die Winkel in $(P) \approx u$ erhalten, die von einer gegebenen Größe $\varphi$ sind und Winkeln won der Größe $\varphi$ bzw. $\pi-\varphi$ in $\left(P^{\prime}\right)$ entsprechen, hat man nur denjenigen mit $c$ konzentrischen Kreis zu konstruieren, dessen Taingenten auf c den mit $p$ äquivalenten Bogen abschneiden. Es gibt in $T$ zweì reelle Lösungen für $z P z^{*}<\varphi<\pi / 2\left(z, z^{*}\right.$ die Doppelpunkte von $S$ auf $\left.c\right)$, in $S$ fur $0 \leqq \varphi<\pi / 2$. Für einen beliebigen Winkel $\psi=\Lambda(p, q)<\pi / 2$ in $(P)$, der von den Halbstrahlen $p, q$ gebildet wird und keinen Rechtswinkelstrahl im Innern enthält (auf welehen Fall die Frage, wie man leicht sieht, immer zurückgeführt werden kann) gilt $\psi \sum \psi^{\prime}\left(\psi^{\prime}\right.$ der entsprechende Winkel in $\left(P^{\prime}\right)$ ), je nachdem $r(q)\} v\left(p^{*}\right)$ ist. Um den Sinn dex Vexänderung eines Winkels bei der Transformation (z. B. bei einer Parallelprojektion) zu 
erhalten, hat man also die Involution $\mathcal{S}$ zu bestimmen und den Drehungssinn eines Halbstrahles bei der Variation von $v$ zu deuten.

Wir hatten oben die Geraden $l_{s}, l_{t}$ betrachtet, die den Kreis $\varkappa_{s}$ in den Punkten $r, s$ schneiden. Die Diagonalpunkte des Vierecks $s_{1} s_{z} r_{1} r_{2}$ sind $S, T, W$, wenn die drei Involutionen $S, T, W$ an $\varkappa_{s}$ dargestellt werden. Stellt man num umgekehrt die Aufgabe, bei gegebenen Kreisen $\varkappa_{s}, \varkappa_{t}$, zu einem gegebenen derartigen Dreieck $S T W$ das Viereck $s_{i} r_{i}$ zu bestimmen, so wird sie offenbar darauf zurückgeführt, in zwei ähnlichen Punktreihen auf $S T$ die zu $S$ und $T$ harmonischen Paare aufzusuchen. Dies ist also die an die oben angestellten Betrachtungen sich anschließende Bestimmung der mit $(P)$ affinen und mit einem gegebenen Systeme $\left(P_{0}^{\prime}\right)$ ähnlichen Systeme $\left(P^{\prime}\right)$, die in $(P)$ in der gegebenen Richtung $q$ parallelprojizient werden können. 